\title{
A Family with Mental Retardation, Epilepsy and Cerebellar Hypoplasia Showing Linkage to Chromosome 20p11.21-q11.23
}

\author{
Fatih Bayrakli $^{\text {b }}$ Mehmet Canpolat ${ }^{d}$ Huseyin Per ${ }^{d}$ Hakan Gumus ${ }^{d}$ \\ Sefer Kumandas ${ }^{d}$ Ugur Kartal $^{a}$ Hatice Balaban ${ }^{c}$ \\ ${ }^{a}$ Neurogenetic Research Laboratory at ${ }^{b}$ Department of Neurosurgery, and ${ }^{c}$ Department of \\ Neurology, Cumhuriyet University School of Medicine, Sivas, and ${ }^{\mathrm{d}}$ Department of \\ Child Neurology, Erciyes University School of Medicine, Kayseri, Turkey
}

\section{Key Words}

Parametric linkage analysis - Cerebellar hypoplasia - Mental retardation · Logarithm of the odds score

\begin{abstract}
Background: Cerebellar hypoplasia $(\mathrm{CH})$ is a rare malformation caused by various etiologies, usually manifesting clinically as nonprogressive cerebellar ataxia with or without mental retardation. The molecular pathogenesis of the autosomal recessive cerebellar ataxias has a wide range of mechanisms. Differential diagnosis and categorization of the recessive cerebellar ataxias, however, need more specific, biochemical and genetic investigation. Methods: This study applied whole-genome linkage analysis to study a family with nonprogressive cerebellar ataxia and additional mental retardation, epilepsy, and facial dysmorphic features. Genotyping and linkage analysis was done using the GeneChip Mapping 250K NspI Array (Affymetrix Inc., Santa Clara, Calif., USA) for genome-wide linkage analysis of the genotyping data from the affected children and their parents. Results: Allegro software version 1.2 was used for multipoint linkage analysis. We assumed an autosomal recessive inheritance pattern and assigned a penetrance of 0.999. Single-nucleotide polymorphism allele frequencies were estimated from the Affymetrix data of the Caucasian family studied. Using these parameters, a theoretical maximum logarithm of the odds score of 2.69 was identified at chromosome 20p11.21-q11.23. Conclusions: This chromosomal locus is unprecedented in autosomal recessive and nonprogressive ataxia disorder. Further investigation might reveal a new causative gene generating the $\mathrm{CH}$ phenotype.
\end{abstract}


Bayrakli et al:: A Family with Mental Retardation, Epilepsy and Cerebellar Hypoplasia Showing Linkage to Chromosome 20p11.21-q11.23

\section{Introduction}

The cerebellum is the control center of the central nervous system for muscle tone, balance, and the coordination of movements. Numerous cerebellar malformations that exist in humans share the prototypic feature of impaired walking with a lack of coordination of the gait and limbs. Cerebellar hypoplasia $(\mathrm{CH})$ is a rare malformation caused by a variety of etiologies. It usually manifests clinically as progressive or nonprogressive cerebellar ataxia with or without mental retardation. Cerebellar ataxia is accompanied by additional clinical features [1-3].

Hereditary ataxias can be divided into autosomal dominant, autosomal recessive, Xlinked, and mitochondrial subtypes, based on the mode of inheritance. Large consanguineous families have been used to map genetic loci for familial forms of autosomal recessive $\mathrm{CH}$ and ataxias [1, 3-5].

In our paper, we describe a Turkish family with cerebellar ataxia, mental retardation, epilepsy, and a recessive trait that was accompanied by facial anomalies, showing a linkage to chromosome 20. This is unprecedented in autosomal recessive cerebellar ataxias.

\section{Methods}

The study was approved by the Cumhuriyet University Committee of Assessment of Scientific Research (protocol No. 10/181). After informed consent was given by the study participants, blood samples were collected from the parents and their children.

The family was identified in Eastern Turkey after an index case directed the attention to symptoms of epilepsy, mental retardation, and balance disorder in the early stages of life. Clinical testing included magnetic resonance imaging (MRI), the Porteus Maze test, electroencephalography, and routine blood work.

We used GeneChip Mapping 250K NspI Arrays (Affymetrix, Santa Clara, Calif., USA) for genome-wide linkage analysis. Single-nucleotide polymorphism (SNP) genotypes were obtained following the Affymetrix GeneChip Mapping protocol. Using the above-mentioned genome analysis programs, a basic analysis and manipulation of the GeneChip data was undertaken. Allegro software version 1.2 (DeCode Genetics, Inc., Reykjavik, Iceland) was used to perform multipoint linkage analysis. We assumed autosomal recessive inheritance and assigned $99 \%$ penetrance and a phenocopy rate of 0.001 . The allele frequencies for the GeneChip SNPs were obtained from Affymetrix.

\section{Results}

The physical examination of the parents was normal. The 3 affected children had frontal bossing, a high palate (fig. 1), and gait ataxia, and their cerebellar tests were impaired. The patients' ataxias were nonprogressive in the clinical course. Prenatal and perinatal histories were unremarkable. An electroencephalography test revealed epileptogenic activity in the left hemisphere temporal lobe in all the offspring. Cranial MRIs of all affected members revealed CH (fig. 2). The temporal lobes of the 3 children were radiologically normal. All children were using Na valproate $(20 \mathrm{mg} / \mathrm{kg} /$ day $)$ for 14,10 , and 3 years, respectively, and did not have epileptic seizures while using this antiepileptic drug. Patient V-1 underwent the Porteus Maze test at 16 years of age, which revealed heavy mental retardation. Patient V-2 did the same test at 13 years of age, showing a mental age of 6.5 years and an IQ score of 50. 
Bayrakli et al:: A Family with Mental Retardation, Epilepsy and Cerebellar Hypoplasia Showing Linkage to Chromosome 20p11.21-q11.23

Patient V-3 did the test at 4.5 years of age, with a mental age of 2 years and an IQ score of 40. All children were delivered at term and none had a history of in utero exposure to infection, drugs, other teratogens, or any trauma. The unaffected parents did not show any abnormal clinical or radiological findings. All children were born to consanguineous parents (fig. 3a).

GeneChip Mapping 250K NspI SNP Arrays (Affymetrix) was used to genotype all available family members (affected offspring and unaffected parents); parametric linkage analysis was performed using Allegro software version 1.2 [6]. We specified complete penetrance of a rare disease allele with a frequency of 0.001 . Using these parameters, a theoretic maximum logarithm of the odds (LOD) score of 2.69 was identified at chromosome 20p11.21-q11.23 (fig. $3 \mathrm{~b}$ ), and a linkage interval of nearly $13 \mathrm{Mb}$ containing more than 100 genes was found.

\section{Discussion}

Differential diagnosis of cerebellar atrophy (CA) and $\mathrm{CH}$ is often very difficult. CA is defined as a cerebellum with initially normal structures in a posterior fossa of normal size, which displays enlarged fissures (interfolial spaces) in comparison to the foliae secondary to tissue loss. $\mathrm{CH}$, however, indicates a compact cerebellum with a reduced volume; while the cerebellar shape is normal, the cerebellar structures are not filling a normally configured posterior fossa, or a small cerebellum is found within a small posterior fossa [7]. As the clinical symptoms of the family studied here were nonprogressive, $\mathrm{CH}$ was the correct diagnosis in this case.

In addition to the radiological morphology of the cerebellum and the progressive or static nature of the disease, the pattern of inheritance and other phenotypic features help differentiating between the ataxic and cerebellar syndromes involved. Most autosomal recessive ataxias are early-onset ataxias traditional classification systems define as ataxias before the age of 20 years [3]. In our family, the symptoms started in early childhood.

Fogel and Perlman [3] categorized autosomal recessive ataxias as either resembling a Friedreich's ataxia phenotype or as having an early-onset phenotype with CA. They also emphasized that due to the heterogeneity among these disorders, further differentiation would generally require a detailed assessment of the phenotype as well as additional diagnostic studies, particularly neuroimaging. Our family phenotype included nonprogressive ataxia with $\mathrm{CH}$, mental retardation, epilepsy, and mild facial anomalies.

The genome-wide linkage analysis of the family localized the linkage interval at chromosome 20p11.21-q11.23. The area was nearly $13 \mathrm{Mb}$ and was flanked by the SNP markers rs1028524 and rs6022147. Our LOD score of 2.69 represented the maximum value obtainable with this particular family.

Our linkage report that associated chromosome 20p11.21-q11.23 with autosomal recessive CA, epilepsy, mental retardation, and other mild facial anomalies was unique and illustrated the genetic heterogeneity of these symptoms. Our LOD score provided suggestive evidence of linkage to this locus, and our linkage interval was a gene-rich region that harbored more than 100 genes listed in the UCSC database. Further investigations of this linkage area will possibly identify a gene that causes these symptoms and phenotypic characteristics. Further investigation using exome sequencing will be helpful in discovering the causative gene of this syndrome. 
Acknowledgment

This work was supported by the Scientific Research Project Fund of Cumhuriyet University under the project No. T-455

\section{Disclosure Statement}

None of the authors has financial or any other conflicts of interest to disclose.

\section{References}

1 Glass HC, Boycott KM, Adams C, et al: Autosomal recessive cerebellar hypoplasia in the Hutterite population. Dev Med Child Neurol 2005;47:691-695.

-2 Turkmen S, Hoffmann K, Demirhan 0: Cerebellar hypoplasia, with quadrupedal locomotion, caused by mutations in the very low-density lipoprotein receptor gene. Eur J Hum Genet 2008;16:1070-1074.

-3 Fogel BL, Perlman S: Clinical features and molecular genetics of autosomal recessive cerebellar ataxias. Lancet Neurol 2007;6:245-257.

4 Vermeer S, Hoischen A, Meijer RP, et al: Targeted next-generation sequencing of a $12.5 \mathrm{Mb}$ homozygous region reveals ANO10 mutations in patients with autosomal-recessive cerebellar ataxia. Am J Hum Genet 2010;87:813-819.

5 Nicolas E, Poitelon Y, Chouery E, et al: CAMOS, a nonprogressive, autosomal recessive, congenital cerebellar ataxia, is caused by a mutant zinc-finger protein, ZNF592. Eur J Hum Genet 2010;18:1107-1113.

-6 Gudbjartsson DF, Jonasson K, Frigge ML, Kong A: Allegro, a new computer program for multipoint linkage analysis. Nat Genet 2000;25:12-13.

-7 Poretti A, Wolf NI, Boltshauser E: Differential diagnosis of cerebellar atrophy in childhood. Eur J Paediatr Neurol 2008;12:155-167.

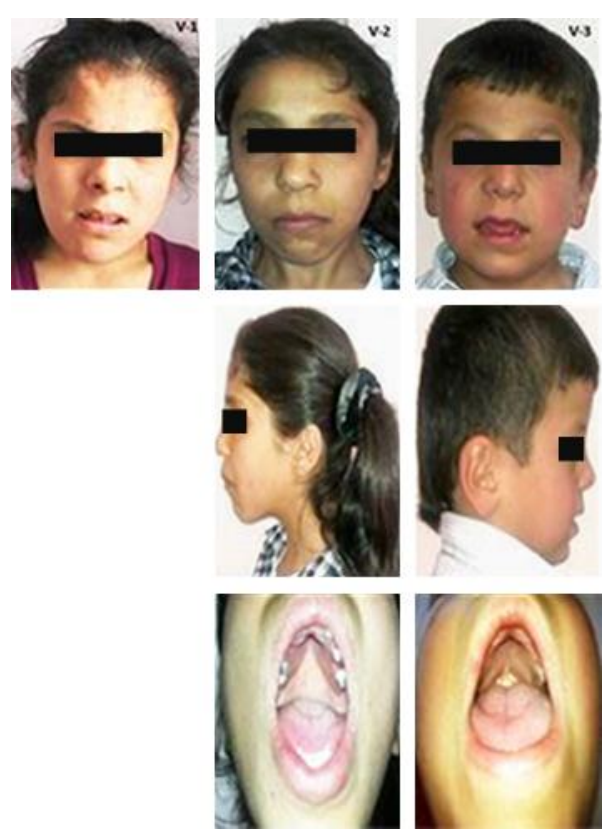

Fig. 1. Facial photographs of the pediatric patients showing a high palate and frontal bossing. 
Bayrakli et al.: A Family with Mental Retardation, Epilepsy and Cerebellar Hypoplasia Showing Linkage to Chromosome 20p11.21-q11.23
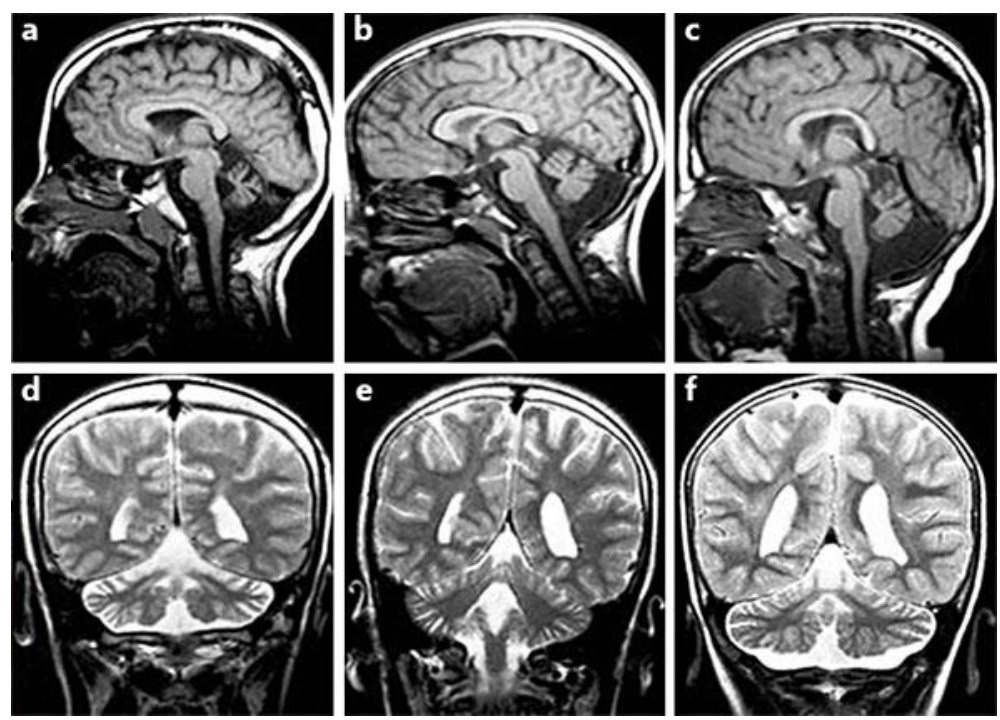

Fig. 2. $\mathrm{T}_{1}$-weighted, midsagittal MRIs of $\mathrm{V}-1$ (a), $\mathrm{V}-2$ (b), and V-3 (c) demonstrating prominent CH. Coronal images of V-1 (d), V-2 (e), and V-3 (f) revealing widened interfolia spaces in the cerebellar hemispheres of the patients.

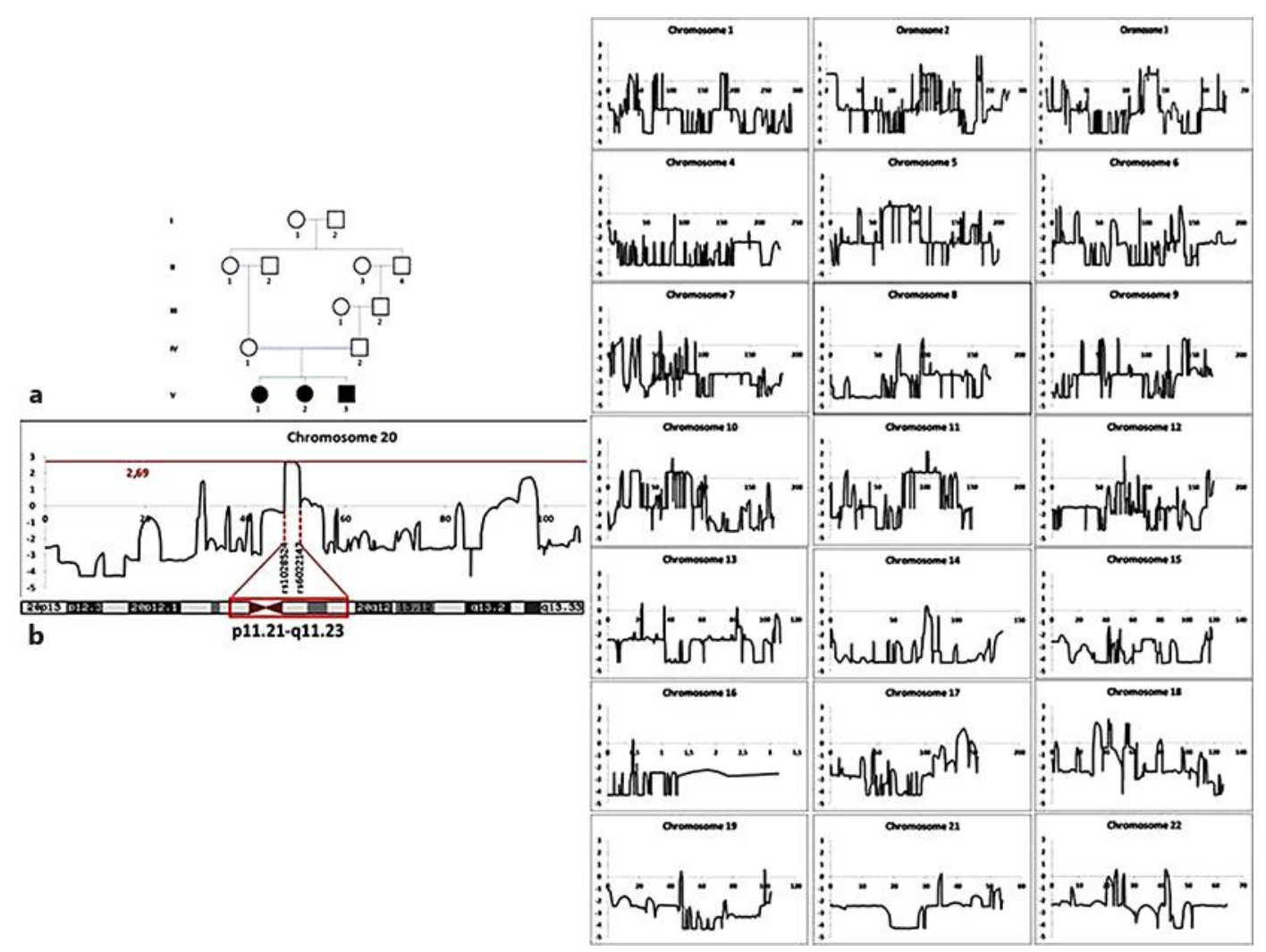

Fig. 3. a Pedigree of the family. Affected members are identified by filled symbols. The locus on chromosome 20 was the only one to give the theoretical maximum for this pedigree (b). Right column: genome-wide linkage analysis of the pedigree with GeneChip Mapping 250K NspI Arrays. The y-axis corresponds to the LOD score, and the $\mathrm{x}$-axis represents distance in $\mathrm{cM}$. 